\title{
Dosimetric Validation of Commissioning Data Validation of Xio Treatment Planning System on a Philips Linear Accelerator
}

\author{
Ahmed Mohamed Hussein Mohamed ${ }^{1 *}$, Ismail Emam ${ }^{2}$ and Amer Mohamed ${ }^{3}$ \\ ${ }^{1}$ Department of Radiation Oncology, Aswan Oncology Institute, Egypt \\ ${ }^{2}$ Department of Physics, Hermitage Medical Clinic Dublin, Ireland \\ ${ }^{3}$ Faculty of Science, Department of Physics, Minia University, Egypt
}

\begin{abstract}
Treatment planning is one of the main steps in radiotherapy. It includes dose, isodose and monitor units (MUs) calculations. The dose calculation is based on algorithms implemented in the treatment planning system (TPS). For a suitable clinical use, these algorithms must calculate the dose as accurately as possible. The aim of this work is the assessment of treatment planning system installed in Aswan Oncology Institute to perform dosimetric validation of various parameters.

Measurements have been performed using existing Elekta linear accelerator, (scanditronix-wellhofer dosimetry) system, and water phantom. A variety of 3D conformal fields were created in Xio TPS on a combined Polymethyl methacrylate (PMMA) phantom and validated against measurements with a calibrated ion chamber. Some of the parameters such as (Tissue phantom ratio (TPR), surface dose, buildup, flatness, symmetry, penumbra, contamination dose) varied including source to surface distance, field size, gantry angle, and depth for all photon and electron energies. The difference between measurements and calculation of flatness and symmetry values at different electron energies were between $-0.4 \%$ to $1.7 \%$ and $6 \mathrm{MV}$ didn't exceed $\pm 0.8 \%$. The mean difference in value of penumbra of electron beams was $-4.97 \%$ and $6 \mathrm{MV}$ was $\pm 5 \%$. TPR and surface dose at $6 \mathrm{MV}$ increased with the field size (FS) increasing. All the resulted difference of measurements and calculation were in agreement with IAEA-TRS430 and Venselaar et al. which didn't exceed $\pm 2 \%$ at flatness, symmetry and $\pm 15 \%$ at penumbra. This investigation on dosimetric validation ensures accuracy of Xio TPS thereby improving patient safety.
\end{abstract}

Keywords: Flatness; Radiotherapy; Penumbra; Dosimetry; Surface dose; Build up

\section{Introduction}

Radiotherapy physics is very important for clinical response of tumours and normal tissues exposed to photon beams and electron beams. The treatment planning systems use computation methods to determine dose distribution in patients from external photon beams and electron beams. Advance algorithm is needed in order to achieve quick and accurate calculation of dose distribution for radiation beams. Depending on treatment modality, an optimum algorithm should be selected. Dose calculation algorithms are the most critical software component in a computerized Treatment Planning System. These modules are responsible for the representation correct of dose in patient, and maybe linked to beam time or monitor unit (MU) calculations.

The dose calculation is based on algorithms implemented in the treatment planning system (TPS). For a suitable clinical use, these algorithms must calculate the dose as accurately as possible. The radiotherapy department at Aswan Oncology Institute combines the Clarkson algorithm and the Pencil Beam (PB) algorithm to measurement. It was necessary to verify that algorithm will not introduce the unexpected results in the clinical practice. So, this study is made to compare the two algorithms with measurements (wellhoferdosimetry system) and reference protocol. The comparisons between algorithms and measurements include from where Beam quality, Surface dose, build-up, flatness, symmetry, penumbra, Contamination dose, buildup region and Absolute dose at different energy (6 MV, 4 $\mathrm{MeV}, 6 \mathrm{MeV}, 8 \mathrm{MeV}, 10 \mathrm{MeV}, 12 \mathrm{MeV}$, and $15 \mathrm{MeV}$ ) and different field size.

\section{Materials and Methods}

In the present study, a Philips-Elekta SL-15 linear accelerator is used. It has two independent tungsten jaws with maximum field size of $40 \mathrm{~cm}$. It provides two photon energies: 6 and $10 \mathrm{MV}$. The distance from the target to the $(\mathrm{X})$ jaws isSource Collimate Distance $(\mathrm{SCDX})=40.9 \mathrm{~cm}$ and $(\mathrm{SCD} y)=27.6 \mathrm{~cm}$ for the $(\mathrm{Y})$ jaws. The flattening filter is located at a distance $(\mathrm{SED})=15.8 \mathrm{~cm}$ from the target (Vendor reference manual) $[1,2]$. These parameters are used to model the Treatment Machine in the Elekta Xio TPS.

Measurements have been performed using the Scanditronixwellhofer relative and absolute dosimetry system [3]. Percentage depth dose (PDD) and beam profiles at $6 \mathrm{MV}$ and available electron beams (4 MeV, $6 \mathrm{MeV}, 8 \mathrm{MeV}, 10 \mathrm{MeV}, 12 \mathrm{MeV}$, and $15 \mathrm{MeV}$ ), TPR then calculated, mean energy, surface dose, Symmetry, Flatness, Penumbra, build up region and contamination dose are evaluated.

\section{Dosimetric measurement tools}

Beam data were acquired using a computer-controlled radiation field analyzer (Scanditronix-Wellhofer AB, Sweden), a water phantom having the scanning area of dimensions $495 \mathrm{~mm} \times 495 \mathrm{~mm} \times 495$

*Corresponding authors: Ahmed Mohamed Hussein Mohamed, Department of Radiation Oncology, Aswan Oncology Institute, Egypt, Tel: 20972303374; E-mail: Prof_ahmed147@yahoo.com

Received January 24, 2018; Accepted February 19, 2018; Published February 26, 2018

Citation: Mohamed AMH, Emam I, Mohamed A (2018) Dosimetric Validation of Commissioning Data Validation of Xio Treatment Planning System on a Philips Linear Accelerator. J Cancer Sci Ther 10: 036-040. doi:10.4172/19485956.1000514

Copyright: @ 2018 Mohamed AMH, et al. This is an open-access article distributed under the terms of the Creative Commons Attribution License, which permits unrestricted use, distribution, and reproduction in any medium, provided the original author and source are credited. 
$\mathrm{mm}(\mathrm{X} / \mathrm{Y} / \mathrm{Z})$ and positional accuracy of $\pm 0.5 \mathrm{~mm}[4]$. The detectors used in this study were parallel Plate, and CC13 thimble ion chamber (Scanditronix-Wellhofer $\mathrm{AB}$, Sweden) [5,6]. It is recommended to position parallel plate, and thimble ion chambers perpendicular tocentral axis CAX of the beam. OmniPro Accept (Version 6.1) software (Scanditronix-Wellhofer AB, Sweden) was used to control the movement of detectors in water phantom and to analyze the acquired PDD and beam profiles [3].

\section{Experimental setup}

In photon measurements, PDD and beam profiles were acquired using different detectors oriented both in parallel and perpendicular direction to central axis (CAX) of the beam for square fields varying from $5 \mathrm{~cm} \times 5 \mathrm{~cm}$ to $30 \mathrm{~cm} \times 30 \mathrm{~cm}$ defined at $100 \mathrm{~cm}$ source to surface distance (SSD) [7]. At the nominal depth of zero, the effective point of measurement of detectors was positioned parallel to the water surface. In electron measurements $6 \times 6,10 \times 10,14 \times 14$, and $20 \times 20$ applicators were used, defined at $95 \mathrm{~cm} \mathrm{SSD} \mathrm{(Figure} \mathrm{1)}$

\section{Percentage depth dose}

Photon PDDs were normalized at the Depth of $10 \mathrm{Cm}$ (D10) with $10 \mathrm{~cm} \times 10 \mathrm{~cm}$ field after measurement with each detector. Where the electron PDDs were normalized at depth of maximum dose (Zmax) The thimble chamber was positioned accurately in the detector holder and PDDs were obtained by scanning the detector from a depth of 30 $\mathrm{cm}$ to the surface of water tank in discrete of $0.5 \mathrm{~mm}$ steps for all field settings as mentioned in the previous section. The photon percentage $\mathrm{D} 10$, and the value of $Z \max$, and electron $Z \max$ in water were analyzed using thimble ion chamber.

\section{Beam profile}

Beam profiles for different field settings mentioned in experimental setup were measured across the center of the field using thimble ionization chamber in cross-line with a target-to-surface distance of $100 \mathrm{~cm}$. The acquired profiles were normalized at $100 \%$ on the CAX of the beam. Cross-line plane profiles for each field along the center of the beam were acquired at $\mathrm{Zmax}$ for $6 \mathrm{MV}$ photon beam and 6,8,10, and $12 \mathrm{MeV}$ electron beams. The cross-line profiles acquired using various thimble chamber were analyzed to find the variation in flatness, symmetry, and penumbral width (20\% to $80 \%)$.

\section{Absolute dose}

Electron absolute dose was measured using parallel plate ionization chamber and PMMA (Scanditronix-Wellhofer AB, Sweden) in Zmax and $10 \mathrm{~cm}$ under them. IAEA TRS 398 protocol was used to obtain the dose, the ion chamber correction factor $(\mathrm{ND}, \mathrm{w})$ was defined by National Institute of Standards(NIS) using Co-60.

\section{Calculating XIO TPS data}

A QA water phantom was created on XIO TPS. The phantom's dimensions were set up as $40 \times 40 \mathrm{~cm}^{2}$ and the slices step as $1 \mathrm{~cm}$. Dose Profiles and planar doses from the scanned phantom were exported. Beams data for photon and electrons were set up as in previous measurements, in Figure 2a. Dose Profile can be evaluated across a plane and then exported, in Figure $2 b$. The dose profiles were used in absolute Normalization mode. The cross-line profiles were analyzed to find the variation in flatness, symmetry, and penumbral. The parameters data were extracted from the Xio planning system, and compared with the same parametersmeasurements. The Analyzed difference is considered as $(\partial)[8,9]$.

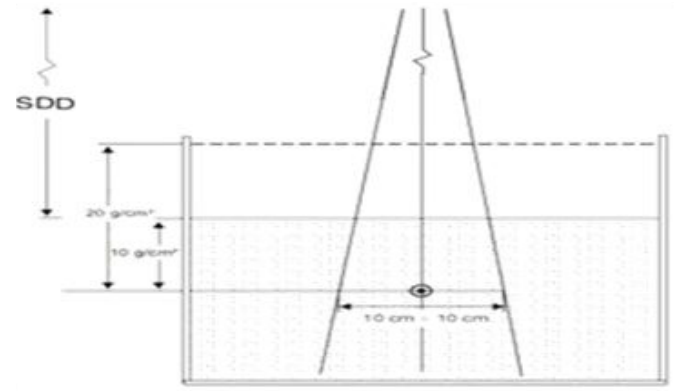

Figure 1: Experimental set-up for the determination of the beam quality index (TPR20,10), PDD, and beam profile.

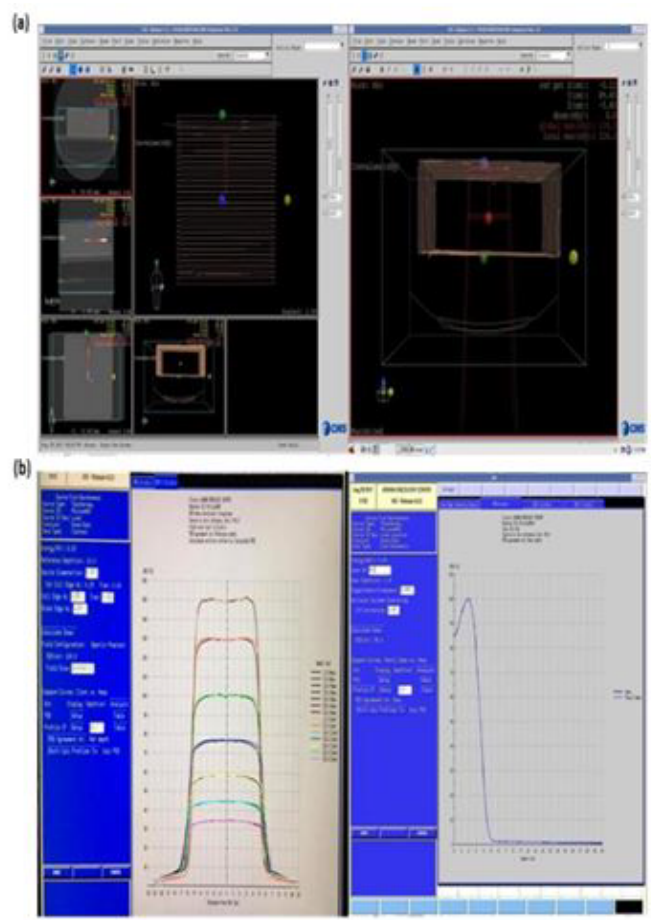

Figure 2: Illustrated - (a) 3-D phantom set up in Xio TPS; (b) Calculation of PDD and beam profile in Xio TPS.

\section{$\partial=100 \%$ (Dcalc.-Dmeas.)/Dmeas.}

${ }^{D}$ calc is the calculated dose at a particular point in the phantom; Dmeasis the measured dose at the same point in the phantom. The difference between measurements and calculationcheck with IAEATRS430 and Venselaar et al. which was Determining the extent of agreement.

\section{Results and Discussion}

One of the major contributions in TPS is the accuracy of dose calculation algorithm. Therefore, it is important to perform various tests to understand the algorithm's limitations. Such tests aim to identify problems and decrease errors in overall patient treatment process. In this study, comparison between dose calculation algorithms (Clarkson and Pencil beam) in common used TPS and measurement (wellhoferdosimetry system) in Aswan Cancer Center was evaluated using TRS430 protocol and Venselaar et al.

The parameters of calculations and measurements are tabulated 

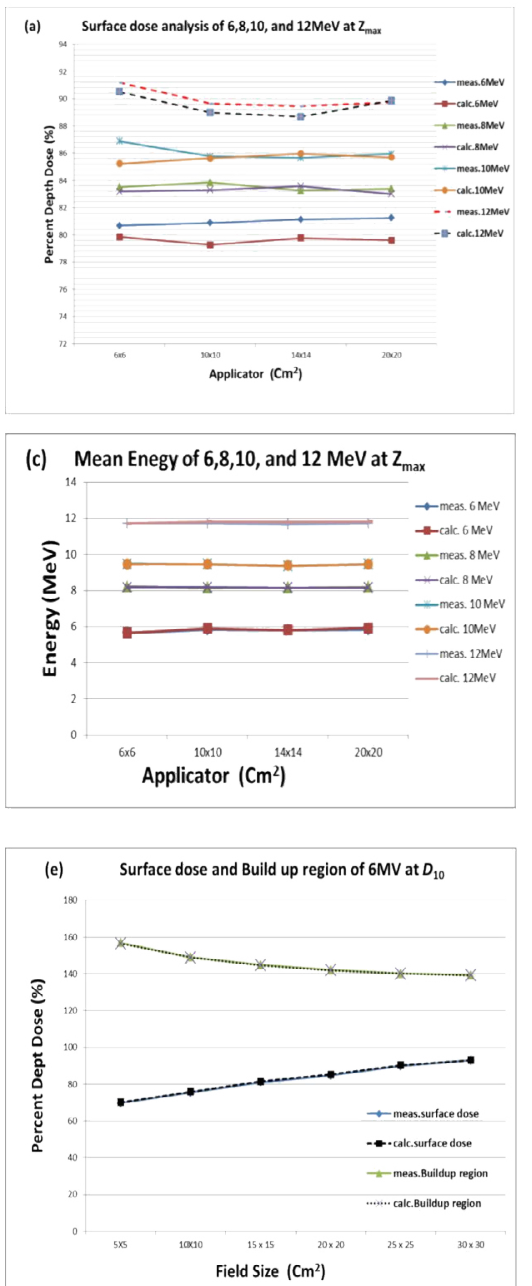
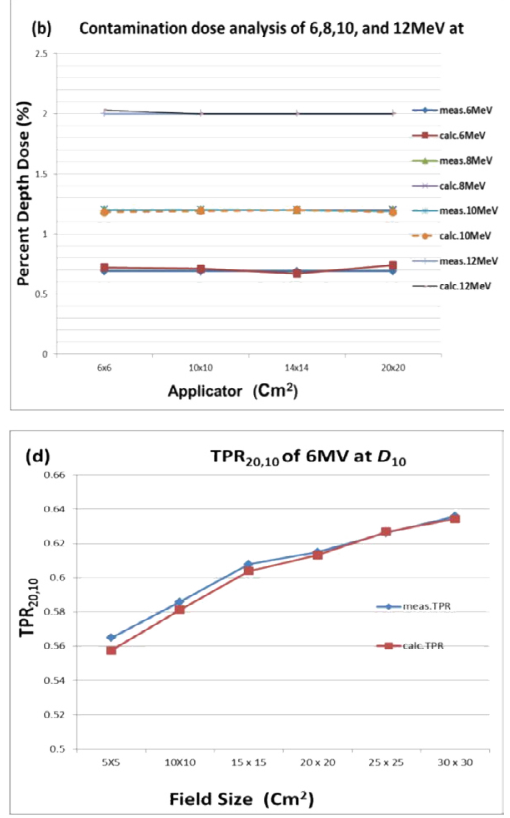

Figure 3: Comparison between measured and calculated by TPS data: (a) surface dose analysis of $6,8,10$, and 12 MeV at $Z$ max (b) contamination dose analysis of 6 , 8,10 , and $12 \mathrm{MeV}$ at $Z_{\max } ;(\mathrm{c})$ mean energy of $6,8,10$, and $12 \mathrm{MeV}$ at $Z_{\max }$; (d) TPR20, 10 of $6 \mathrm{MV}$ at $\mathrm{D}_{10}$; (e) surface dose and build up region of $6,8,10$, and $12 \mathrm{MeV}$ at $D_{10}$, respectively.

in Table 1 for all electron energies at Zmax and applicator $(6 \times 6,10$ $\times 10,14 \times 14$, and $20 \times 20$ ). The symmetry data of calculations and measurements was between $98.29 \%$ to $100.39 \%$, the average values of difference in measurements and calculations for $8 \mathrm{MeV}, 10 \mathrm{MeV}$, and $12 \mathrm{MeV}$ energy equal $0.67 \%, 1 \%, 0.25 \%$, and $0.37 \%$, respectively. But $10 \mathrm{MeV}$ energy at FS $(10 \times 10)$ is low to $0.1 \%, 4 \mathrm{MeV}$ energy at FS $(6$ $\times 6$ and $20 \times 20)$ equal $1 \%$ and FS $(10 \times 10$ and $14 \times 14)$ equal $0.1 \%, 6$ $\mathrm{MeV}$ energy at FS $(6 \times 6$ and $14 \times 14)$ equal $1.7 \%$ and FS $(10 \times 10$ and $20 \times 20$ ) equal $0.2 \%$ (Figure 3 ) (Table 1 ). With regard to the flatness and penumbra, the values of flatness and penumbra for measurements and calculations were $99.98 \%$ to $100.92 \%, 0.86 \mathrm{Cm}$ to $1.25 \mathrm{Cm}$, respectively. The average values of difference in Flatness for $6 \mathrm{MeV}, 8 \mathrm{MeV}, 10 \mathrm{MeV}$, and $12 \mathrm{MeV}$ energies equal $-0.2 \%,-0.1 \%,-0.23 \%$, and -0.43 , respectively. $8 \mathrm{MeV}$ energy at FS $(10 \times 10)$, the difference value was up to $-0.9 \%$. The average values of difference in Penumbra for $6 \mathrm{MeV}, 8 \mathrm{MeV}, 10 \mathrm{MeV}$, and $12 \mathrm{MeV}$ energies equal $-8.93 \%,-9.2 \%,-8.95 \%$, and $-4.8 \%$, respectively. But $6 \mathrm{MeV}$ energy at FS $(20 \times 20)$, the value was even to $2.4 \%$ (Table 2 ). All difference in symmetry and Flatness between measurement and calculation of all electron energies were between $-0.4 \%$ to $1.7 \%$ and all difference didn't Exceed $\pm 2 \%$. As for penumbra, the difference was between $-9.8 \%$ to $2.4 \%$ and all difference didn't Exceed $\pm 15 \%$ (Table 3). The absolute dose data of measurements and calculations was tabulated in Table 2 at FS $(10 \times 10)$ for all electron energies. The absolute dose of measurements and calculations was between $99.26 \%$ to $101.41 \%$ and the difference of absolute dose between them was in $\pm 2 \%$. When looking at other parameters such as (surface dose, mean energy and contamination dose) of all electron energies at $\mathrm{Zmax}$ and applicators $(6 \times 6,10 \times 10,14 \times 14$ and $20 \times 20)$ were illustrated in Figure 3. Shown in Figure 3a was comparison of surface dose values between measurements and calculations of all electrons, the surface dose of measurements and calculations at $6 \mathrm{MeV}, 8 \mathrm{MeV}, 10 \mathrm{MeV}$, and $12 \mathrm{MeV}$ was 79.2 to $81.2,82.8$ to $84,85.2$ to 86.8 , and 89.2 to 91.6 , respectively. The surface dose difference between measurements and calculations for all applicators, maximum difference of $12 \mathrm{MeV}$ were observed in the smaller FS and minimum differences were observed in the larger FS, at $6 \mathrm{MeV}, 8 \mathrm{MeV}$, and $10 \mathrm{MeV}$ were close to each other. But the difference of $10 \mathrm{MeV}$ at FS $6 \mathrm{~cm} \times 6 \mathrm{~cm}$ raised. In Figure $3 \mathrm{~b}$, the contamination dose of measurement and calculation at $6 \mathrm{MeV}$ was below $1 \%, 8 \mathrm{MeV}$ and $10 \mathrm{MeV}$ between $1 \%$ to $1.2 \%$ and $12 \mathrm{MeV}$ was close to $2 \%$. The dose in a patient is contributed by bremsstrahlung interactions of electrons with the collimation system (scattering foils, chambers, collimator jaws, etc.) [3]. This photon contamination creates various doses in electron beams. Typical $\mathrm{x}$-ray contamination doseto a patient ranges from approximately $0.5 \%$ to $1 \%$ in the energy range of 6 


\begin{tabular}{|c|c|c|c|c|c|c|c|c|c|c|c|c|c|}
\hline \multirow{2}{*}{ Type } & \multirow{2}{*}{ Applicator } & \multicolumn{3}{|c|}{$6 \mathrm{MeV}$} & \multicolumn{3}{|c|}{$8 \mathrm{MeV}$} & \multicolumn{3}{|c|}{$10 \mathrm{MeV}$} & \multicolumn{3}{|c|}{$12 \mathrm{MeV}$} \\
\hline & & Meas.\% & Calc.\% & Diff \% & Meas. $\%$ & Calc.\% & Diff \% & Meas.\% & Calc.\% & Diff \% & Meas.\% & Calc.\% & Diff \% \\
\hline \multirow{4}{*}{ Flatness } & $6 \times 6$ & 100.36 & 99.98 & -0.4 & 100.16 & 100 & -0.2 & 100.12 & 99.99 & -0.1 & 100.09 & 100 & -0.1 \\
\hline & $10 \times 10$ & 100.17 & 100 & -0.2 & 100.92 & 100 & -0.9 & 100.29 & 100 & -0.3 & 100.38 & 100 & -0.4 \\
\hline & $14 \times 14$ & 100.07 & 100 & -0.1 & 100.05 & 100 & 0.0 & 100.06 & 100 & -0.1 & 100.82 & 100 & -0.8 \\
\hline & $20 \times 20$ & 100.12 & 100 & -0.1 & 100.10 & 100 & -0.1 & 100.41 & 100 & -0.4 & 100.39 & 100 & -0.4 \\
\hline \multirow{4}{*}{ Symmetry } & $6 \times 6$ & 98.29 & 99.93 & 1.7 & 99.38 & 99.75 & 0.4 & 99.1 & 100.08 & 1.0 & 99.5 & 100.03 & 0.5 \\
\hline & $10 \times 10$ & 99.9 & 100 & 0.1 & 99.50 & 100 & 0.5 & 100.1 & 100.01 & -0.1 & 100.1 & 99.97 & -0.1 \\
\hline & $14 \times 14$ & 99.01 & 99.98 & 1.0 & 99.11 & 100 & 0.9 & 99.2 & 100.06 & 0.9 & 99.78 & 100.39 & 0.6 \\
\hline & $20 \times 20$ & 99.7 & 100 & 0.3 & 99.1 & 100 & 0.9 & 98.9 & 100 & 1.1 & 100.01 & 99.99 & 0.0 \\
\hline \multirow{4}{*}{ penumbra } & $6 \times 6$ & 0.95 & 0.86 & -9.4 & 1.08 & 0.98 & -9.3 & 1.19 & 1.08 & -9.2 & 1.05 & 1.03 & -1.9 \\
\hline & $10 \times 10$ & 0.97 & 0.89 & -8.2 & 1.07 & 0.97 & -9.3 & 1.2 & 1.11 & -7.5 & 1.08 & 1.02 & -5.6 \\
\hline & $14 \times 14$ & 0.98 & 0.89 & -9.18 & 1.05 & 0.96 & -8.6 & 1.22 & 1.1 & -9.8 & 1.15 & 1.07 & -7.0 \\
\hline & $20 \times 20$ & 0.85 & 0.87 & 2.4 & 1.03 & 0.93 & -9.7 & 1.18 & 1.07 & -9.3 & 1.25 & 1.19 & -4.8 \\
\hline
\end{tabular}

Table 1: Comparison of values between measurements and calculations at some parameter for all electron energies.

\begin{tabular}{|c|c|c|c|c|c|c|c|}
\hline Energy & Depth & SSD & PDD & $\mathbf{N}_{\mathrm{DW}}$ & Dose meas. & Dose calc. & Diff. \% \\
\hline $4 \mathrm{mev}$ & 0.9 PMMA & 100 & 98 & 5.141 & 101.19 & 100 & -1.1 \\
\hline $6 m e v$ & 1.1 PMMA & 100 & 99.3 & 5.141 & 101.08 & 100 & -1.06 \\
\hline $08 \mathrm{Mev}$ & 1.6 PMMA & 100 & 100 & 5.141 & 99.26 & 100 & 0.74 \\
\hline $10 \mathrm{Mev}$ & 1.8 PMMA & 100 & 100 & 5.141 & 100.74 & 100 & -0.73 \\
\hline $12 \mathrm{Mev}$ & 2.1 PMMA & 100 & 100 & 5.141 & 101.41 & 100 & -1.4 \\
\hline $15 \mathrm{Mev}$ & 2.8 PMMA & 100 & 99.9 & 5.141 & 101.17 & 100 & -1.2 \\
\hline
\end{tabular}

Table 2: Shows parameters and absolute dose for all electron energies at $10 \times 10$ field size.

\begin{tabular}{|c|c|c|c|c|c|c|c|c|c|}
\hline \multirow{2}{*}{ Field size } & \multicolumn{3}{|c|}{ Flatness } & \multicolumn{3}{|c|}{ Symmetry } & \multicolumn{3}{|c|}{ Penumbra } \\
\hline & Meas. $\%$ & Calc.\% & Diff \% & Meas. $\%$ & Calc.\% & Diff \% & Meas. & Calc. & Diff \% \\
\hline $5 \times 5$ & 100.47 & 100.16 & -0.3 & 100.39 & 100.09 & -0.3 & 0.66 & 0.7 & 6.1 \\
\hline $10 \times 10$ & 100.97 & 100.56 & -0.4 & 100.32 & 100.02 & -0.3 & 0.72 & 0.75 & 4.2 \\
\hline $15 \times 15$ & 100.88 & 100.93 & 0.05 & 99.58 & 99.97 & 0.4 & 0.78 & 0.72 & -7.7 \\
\hline $20 \times 20$ & 100.98 & 100.94 & $0.04-$ & 99.75 & 100 & 0.3 & 0.73 & 0.7 & -4.1 \\
\hline $25 \times 25$ & 101.45 & 101.46 & 0.01 & 99.58 & 99.98 & 0.4 & 0.75 & 0.73 & -2.7 \\
\hline $30 \times 30$ & 101.68 & 101.61 & -0.1 & 99.17 & 100 & 0.8 & 0.69 & 0.72 & 4.7 \\
\hline
\end{tabular}

Table 3: Comparison of values between measurements and calculations at some parameter for $6 \mathrm{MV}$.

to $12 \mathrm{MeV} ; 1 \%$ to $2 \%$, from 12 to $15 \mathrm{MeV}$; and $2 \%$ to $5 \%$, from 15 to 20 $\mathrm{MeV}[10,11]$. The difference between measurement and calculation was venial. Figure $3 \mathrm{c}$ shows that the difference between measurement and calculation at energy of electron beam was venial. The values of electron energies were very near to required value for energy. TPS calculations and measurements were yielded to some test toward photon which were tabulated in Table 3 and figured in Figure 2 at Zmax and different field size. In Table 3 the values of measurements and calculations of flatness, symmetry and penumbra were from $100.16 \%$ to $101.68 \%, 99.17 \%$ to $100.39 \%$, and $0.66 \mathrm{~cm}$ to $0.78 \mathrm{~cm}$, respectively. The difference values of flatness between measurement and calculation were from $-0.04 \%$ to $0.05 \%$, symmetry was from $-0.3 \%$ to $0.8 \%$ and penumbra was $-7.7 \%$ to $6.1 \%$. In Figure $3 \mathrm{~d}$, the TPR was between 0.56 to 0.64 , it is small in small field size and it increases with increasing field size, due to Challenges with small field dosimetry including lack of charge particle equilibrium, partial volume averaging, and positioning accuracy [12]. The difference between measurement and calculation was maximum in small field size and minimum in large field size. Figure $3 e$ shows, the surface dose and build-up region of measurements and calculations at $\mathrm{Zmax}$ for $6 \mathrm{MV}$, surface dose values increase with increasing field size, Due to Photons scattered from the collimators, flattening filter, air and High-energy electrons produced by photon interactions in air [1]. The difference of surface dose between measurement and calculation was slight. The dose of measurements and calculations in build-up region was large in small field size and small in large field size and the difference between measurement and calculation was very small.

\section{Conclusion}

All results of difference of flatness, symmetry penumbra and contamination at $6 \mathrm{MV}$ and electron beams were less than $\pm 2 \%, \pm$ $2 \%, \pm 15 \%$, and $\pm 30 \%$, respectively. 2.4 TPR and surface dose increase with increasing field size. 1 Thus, Xio TPS commission was successfully verified on dosimtric measurements.

\section{References}

1. Philips $E$ (1997) Accelerator machine reference manual.

2. Philips E (1999) reference manual copyright Elekta Oncology Systems Ltd

3. System Manual to OmniPro-Accept (2001) Version 6.0A, DAA011 90003 01, By Scanditronix-Wellhofer

4. https://www.scribd.com/doc/37993938/Blue-Phantom-Manual

5. Scanditronix-Wellhofer (2000) User's Manual to Thimble Chamber CC13

6. Scanditronix-Wellhofer (2000) User's Manual to parallel plate Chamber PPC

7. IAEA TRS-398 (2000) Absorbed dose determination in external beam radiotherapy: An International code of practice for dosimetry based on standards of absorbed dose to water IAEA, Vienna.

8. Venselaara J, Welleweerd H, Mijnheer B (2001) Tolerances for the accuracy of photon beam dose calculations of treatment planning systems. Radiother Oncol 60: 191-201.

9. IAEA TRS-430 (2004) Commissioning and quality assurance of computerized planning systems for radiation treatment of cancer, International Atomic Energy Agency. 
Citation: Mohamed AMH, Emam I, Mohamed A (2018) Dosimetric Validation of Commissioning Data Validation of Xio Treatment Planning System on a Philips Linear Accelerator. J Cancer Sci Ther 10: 036-040. doi:10.4172/1948-5956.1000514

10. Faiz MK, John PG (2014) The Physics of Radiation Therapy (5th edn) Lippincott Williams \& Wilkins, Wolterskluwer, Netherlands.

11. Narayanasamy G, Cruz W, Papanikolaou N, Stathakis S (2015) Comparison between measured tissue phantom ratio values andcalculated from percent depth doses with and without peakscatter correction factor in a $6 \mathrm{MV}$ beam. Int $\mathrm{J}$ Cancer Ther Onc 2330-4049.

12. Podgorsak EB (2005) Radiation Oncology Physics, A Handbook for teachers and students, International Atomic Energy Agency, Vienna. 